Article

\title{
Langmuir Probe Diagnostics with Optical Emission Spectrometry (OES) for Coaxial Line Microwave Plasma
}

\author{
Chi Chen $₫$, Wenjie Fu*® , Chaoyang Zhang $₫$, Dun Lu $₫$, Meng Han $₫$ and Yang Yan \\ School of Electronic Science and Engineering and Terahertz Science and Technology Key Laboratory of \\ Sichuan Province, University of Electronic Science and Technology of China, Chengdu 610054, China; \\ chenchi03@163.com (C.C.); chaoyangzhang@std.uestc.edu.cn (C.Z.); ludun@std.uestc.edu.cn (D.L.); \\ 201711040132@std.uestc.edu.cn (M.H.); yanyang@uestc.edu.cn (Y.Y.) \\ * Correspondence: fuwenjie@uestc.edu.cn
}

Received: 28 October 2020; Accepted: 12 November 2020; Published: 16 November 2020

Featured Application: Plasma diagnostics with a dirty Langmuir Probe.

\begin{abstract}
The Langmuir probe is a feasible method to measure plasma parameters. However, as the reaction progresses in the discharged plasma, the contamination would be attached to the probe surface and lead to a higher incorrect electron temperature. Then, the electron density cannot be obtained. This paper reports a simple approach to combining the Langmuir probe and the optical emission spectrometry (OES), which can be used to obtain the electron temperature to solve this problem. Even the Langmuir probe is contaminative, the probe current-voltage (I-V) curve with the OES spectra also gives the approximate electron temperature and density. A homemade coaxial line microwave plasma source driven by a $2.45 \mathrm{GHz}$ magnetron was adopted to verify this mothed, and the electron temperature and density in different pressure (40-80 $\mathrm{Pa})$ and microwave power (400-800 W) were measured to verify that it is feasible.
\end{abstract}

Keywords: plasma diagnostics; Langmuir probe; optical emission spectrometry; electron temperature; electron density

\section{Introduction}

In industrial plasma processing, such as microwave plasma chemical vapor deposition (PECVD) [1-3], it would be useful to directly control plasma properties such as electron temperature and density [4]. For plasma diagnostics, the Langmuir is widely used [5,6]. A Langmuir probe means a probe placed in charged plasma. Though measuring the current-voltage (I-V) curve, the plasma's electron temperature and density can be estimated. A lot of Langmuir probe structures have been researched by a lot of institutions around the world to monitor various plasmas.

However, when the Langmuir probe is used to measure plasma for a long time, especially in PECVD, as the plasma reaction proceeds, the Langmuir probe will adsorb impurities and become dirty. Moreover, if the electron temperature and density are obtained by calculating this dirty Langmuir probe $(\mathrm{I}-\mathrm{V})$ curve, the electron temperature and density would be incorrect $[4,7-9]$. The contamination on the probe surface is very common in various probe designs. The contamination has become a significant problem that limits the application of the Langmuir probe.

The optical emission spectrometry (OES) is a non-intrusive plasma diagnostics method, which is easy to performed, and it is also frequently used in studies of plasma [10-12]. Therefore, the plasma reaction cannot bring contamination to the OES. During the plasma reaction, OES can stably measure reliable electron temperature. The Langmuir can measure both the electron temperature and electron 
density. Both electron temperature and electron density are very important for diagnosing plasma. However, as the plasma reaction progresses, the contamination is attached to the probe surface. Most of these clean probe methods need to interrupt the plasma reaction. However, in the experiment, the result shows that the contamination on the Langmuir probe has little effect on the ion saturation current, as reported in other articles $[7,8,13]$. In other words, the ion saturation does not change. Therefore, if the electron temperature Tev can be measured by optical emission spectrometry (OES), the electron density can be calculated from the I-V curve of the probe with contamination. Therefore, combining the Langmuir probe and OES, both electron temperature and density would be measured, even if the probe has been contaminated. In other words, the cleaning interval of the Langmuir probe would be significantly extended.

Based on the above proposal, a verification experiment is carried on and presented in this paper. The experiment has been done in a homemade coaxial line microwave plasma source driven by $2.45 \mathrm{GHz}$ magnetron and using argon as feeding gas. The results show that the contaminative Langmuir probe I-V curve with the OES can give the approximately electron temperature and density. Besides, by measuring the electron temperature and density under different pressures (40-80 $\mathrm{Pa}$ ) and microwave power $(400-800 \mathrm{~W})$, it is proved that this method is feasible.

\section{Experiment Setup}

The schematic diagram of the microwave-generated plasma system used in the experiment is shown in Figure 1.

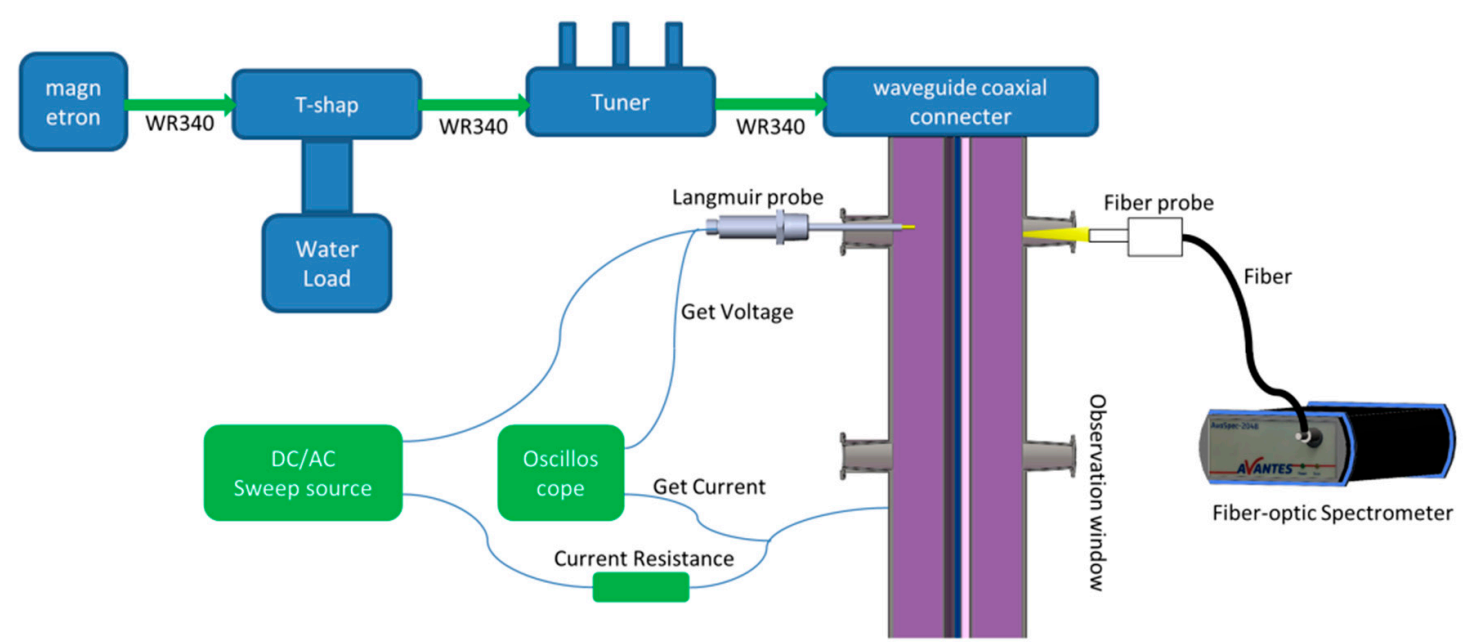

Figure 1. Schematic diagram of the coaxial microwave-driven plasma with Langmuir probe and emission spectroscopy.

The magnetron was used to generate continuous-wave signals at a fixed output frequency of $2.45 \mathrm{GHz}$. The output power of the magnetron varied from 0-800 W. The output wave was launched into the WR340 waveguide using TE10 mode to transfer. An isolation circulator with a water load in an isolated way was connected to the magnetron. A waveguide coaxial connecter was used for waves to enter the coaxial plasma reaction chamber from the WR340 waveguide. An automated, three stobs tuner was used to match the impedance, so that the maximum amount of power could be coupled with the plasma.

This reaction cavity used a coaxial waveguide for microwave transmission. The external diameter of the coaxial waveguide was $150 \mathrm{~mm}$, and its inner conductor was $8 \mathrm{~mm}$. A hollow glass rod was used to surround the coaxial inner diameter. The inner diameter of the glass tube was $29 \mathrm{~mm}$, and the external diameter was $33 \mathrm{~mm}$. Using two O-ring seals to form a vacuum-sealed cavity in the middle of the glass rod's outer wall, both ends of the glass rod were connected to an O-ring seal to form a vacuum-sealed cavity between the glass rod's outer wall and the reaction cavity inner surface. 
There were several KF flanges on the outer wall of the cavity, and the Langmuir probe was mounted on a KF flange, which is shown in detail in Figure 2.

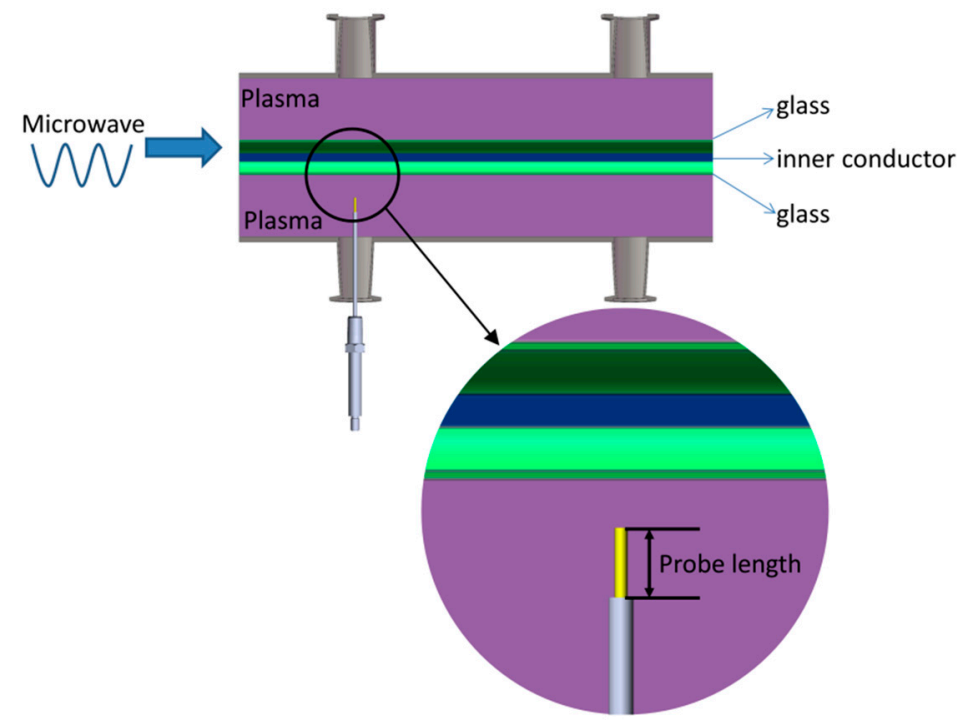

Figure 2. The structure of the coaxial reaction cavity and the Langmuir probe.

In the experiment, firstly, a vacuum pump was used to pump the pressure below $10 \mathrm{~Pa}$. Then, the argon flow rate was adjusted to stabilize the working pressure to $80 \mathrm{~Pa}$. After entering the microwave with $800 \mathrm{~W}$ power, the plasma was discharged, and the light could be observed from the observation window, as shown in Figure 3.

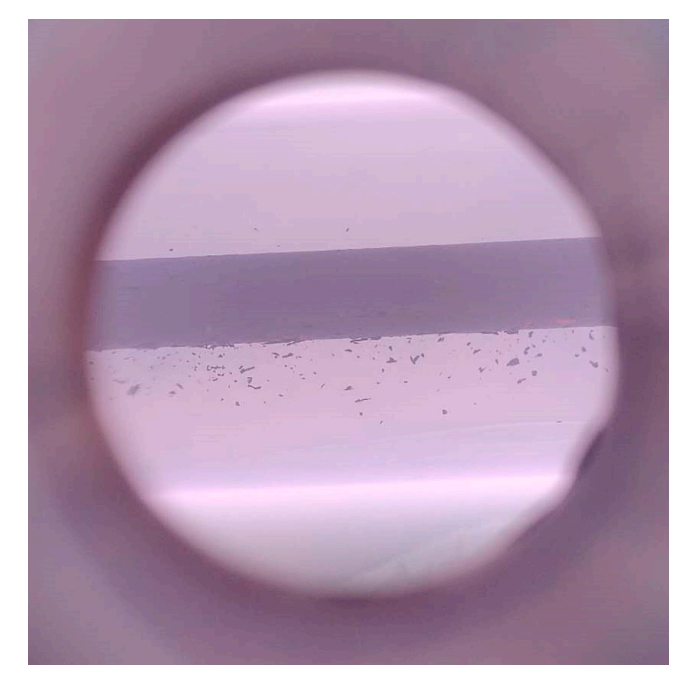

Figure 3. Coaxial line plasma driven by $800 \mathrm{~W}$ microwave at fill pressure of $80 \mathrm{~Pa}$.

\section{The Method of Electron Density and Temperature by Langmuir Probes}

For low-temperature plasmas $(<10 \mathrm{eV})$, the Langmuir probes are generally used to measure the plasma density and electron temperature. A Langmuir probe consists of a bare wire or metal disk, immersed in the plasma, with a sweep voltage to collect electron and ions [5]. In this study, the probe had a $1.0 \mathrm{~mm}$ diameter and was $20 \mathrm{~mm}$ long, suspended in a $12 \mathrm{~mm}$ tube with the vacuum seal at the upper end. The probe tip was a tungsten rod $1 \mathrm{~mm}$ in diameter and $8 \mathrm{~mm}$ long.

When a probe is immersed in plasmas, the current is dependent on the ions current and the electron current. By loading a sweeping voltage on the probe, the I-V curve of the probe is obtained. According to the different current composition, the I-V curve can be divided into three regions [5]: 
(1) Ion saturation region.

(2) Transition region.

(3) Electron saturation region where ions are repelled, but electrons are attracted to the probe.

When a negative voltage is applied on the probe, the positive ions approach to the probe. However, there is an electronic shield on the probe surface, which is the electron Debye length.

The ion saturation region means the probe voltage $(\mathrm{Vp})$ on the probe is sufficiently negative to collect all the ions with the Bohm velocity. The ions are accelerated through a "pre-sheath" to the required energy. When $T_{e} \gg T_{i}$, the ion saturation current is determined by the electron temperature. Therefore, ion saturation current is, approximately, $[7,14-16]$ :

$$
\mathrm{I}_{\text {sat }}=0.605 \mathrm{~A}_{\text {proben }} \sqrt{\frac{\mathrm{kT}}{\mathrm{mi}}}
$$

Here, e is the electron charge, $\mathrm{k}$ is Boltzmann constant, Te is electron temperature, $A_{\text {probe }}$ is the surface area of the probe, $\mathrm{mi}$ is the ion mass. $\mathrm{n}$ is the plasma density in this paper, and we only consider the case where electron density and ion density are equal.

In the transition region, the current consists of both electron current and ions current. Compared to electron current, the ion current is negligible.

The electron current is expressed as follows:

$$
\begin{aligned}
& \mathrm{I}_{\mathrm{e}}=\mathrm{I}_{\mathrm{e} 0} \exp \left[\frac{\mathrm{e}\left(\mathrm{V}_{\mathrm{p}}-\mathrm{V}_{\mathrm{s}}\right)}{\mathrm{k} \mathrm{T}_{\mathrm{e}}}\right] \\
& \mathrm{I}_{\mathrm{e} 0}=\mathrm{ne}\left(\frac{\mathrm{kT} \mathrm{T}_{\mathrm{e}}}{2 \pi \mathrm{m}_{\mathrm{e}}}\right)^{1 / 2} \mathrm{~A}_{\text {probe }}
\end{aligned}
$$

Here, Vs is the space potential, $\mathrm{I}_{\mathrm{e}}$ is the electron current, $\mathrm{I}_{\mathrm{e} 0}$ is the saturation electron current. Equation (4) can be achieved by transforming Equation (2). Equation (4) shows that the slope of the (ln I)-Vp curve is exactly $1 / \mathrm{TeV}$ (which is convenient to write $\mathrm{kTe} / \mathrm{e}$ as $\mathrm{Te} / \mathrm{V}$, the electron temperature in $\mathrm{eV}$ ) and is a good measure of the electron temperature [5,7].

$$
\ln \mathrm{I}_{\mathrm{e}}=\frac{1}{\mathrm{~T}_{\mathrm{ev}}}\left(\mathrm{V}_{\mathrm{p}}-\mathrm{V}_{\mathrm{s}}\right)+\operatorname{lnI}_{\mathrm{e} 0}
$$

Bring the result of Tev into Equation (1), the plasma density (n) can be obtained.

When the plasma is just charged, the I-V curve of the Langmuir probes immersed in coaxial microwave driven plasma is shown in Figure 4, which is measured under the argon fill pressure $\mathrm{Pa}=80 \mathrm{~Pa}$ and microwave power Mpower $=800 \mathrm{~W}$. The applied voltage on Langmuir probes is $\mathrm{AC}$ $60 \mathrm{~V}-50 \mathrm{~Hz}$.

As shown in Figure 4, when the voltage is positive, the voltage curve is consistent with the current curve. When the voltage is negative, the current curve first decreases with the voltage and then becomes smooth at around about $-20 \mathrm{~V}$. This is because $+60 \mathrm{~V}$ does not make the probe current reach the electron saturation region. Therefore, when the voltage increases from $0 \mathrm{~V}$ to $60 \mathrm{~V}$, the current also increases exponentially. When the voltage is less than $-20 \mathrm{~V}$, the ion current variation is very small (less than $0.005 \mathrm{~mA}$ ), and it enters the ion saturation region. Combining the voltage curve and the current curve, the I-V curve of the Langmuir probes containing ion saturation region and transition region can be obtained. 


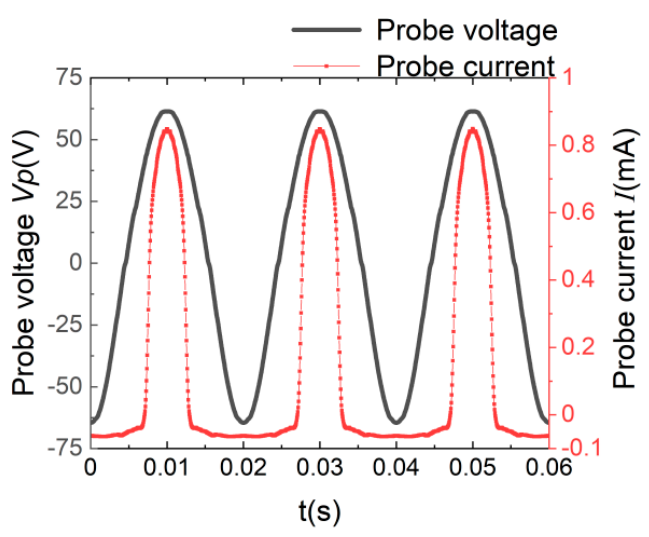

(a)

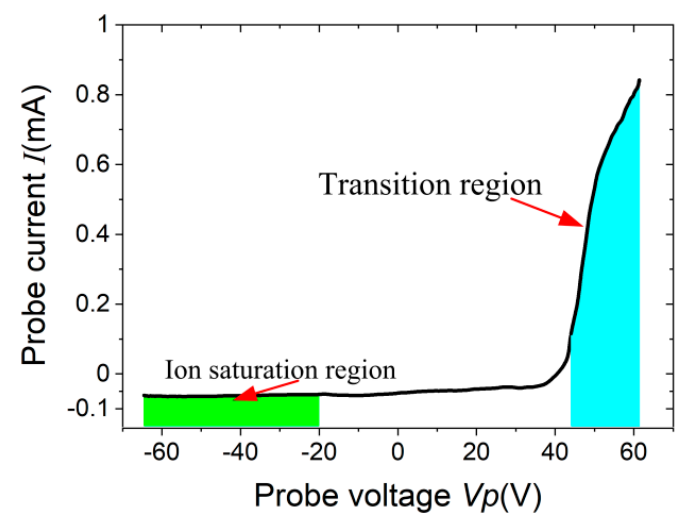

(b)

Figure 4. The I-V curve of the clean Langmuir probe (a) the voltage and current curve, (b) I-V curve.

From the I-V curve of the Langmuir probes, the (ln I-Vp) curve slope in the transition region is 0.736 , namely Tev.probe $=1.3578 \mathrm{eV}$. In addition, the average current in the ion saturation current region is $0.0613 \mathrm{~mA}$, namely Isat $=0.0613 \mathrm{~mA}$. Inserting the parameters in Table 1 into Equation (1), it can be found that the density of the plasma is $n=1.3569 \times 10^{-16} \mathrm{~m}^{-3}$.

Table 1. Parameters of the coaxial microwave driven plasma and Langmuir probes used to measure electron temperature and density.

\begin{tabular}{cccc}
\hline Parameters & Symbol & Value & Units \\
\hline Ion species & $\mathrm{Ar}+$ & & \\
Ion mass & $\mathrm{mi}$ & $6.7 \times 10^{-26}$ & $\mathrm{~kg}$ \\
Microwave power & Mpower & 800 & $\mathrm{~W}$ \\
Pressure & Pa & 80 & $\mathrm{~Pa}$ \\
Probe diameter & dprobe & 1 & $\mathrm{~mm}$ \\
Probe length & lprobe & 8 & $\mathrm{~mm}$ \\
\hline
\end{tabular}

The Langmuir probe is a feasible method to measure the plasma. However, as the plasma reaction progresses, there is gradually some phase difference between the voltage curve and the current curve, and the current is decreased compared with the beginning, as shown in Figure 5.

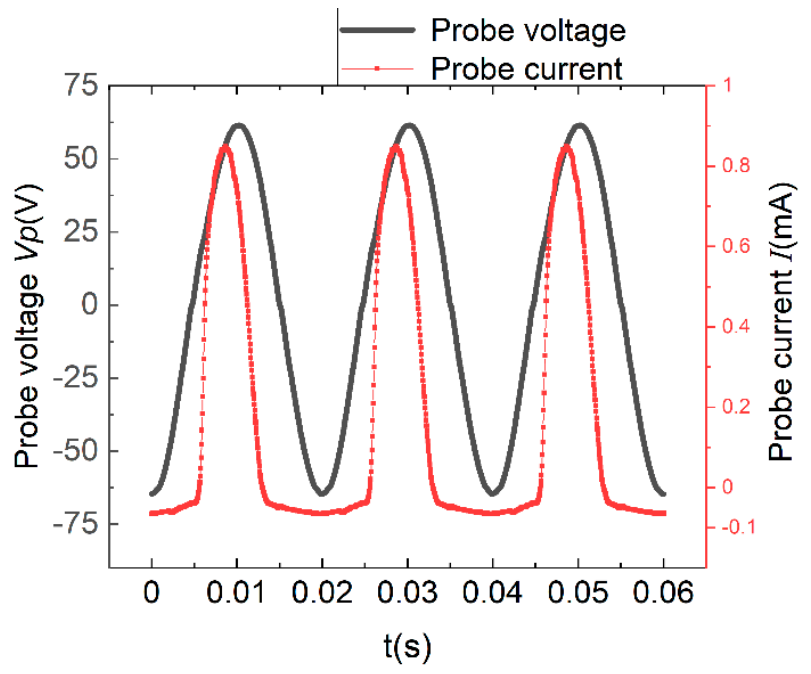

Figure 5. The voltage and current curve of the Langmuir probe with contamination. 
It is generally considered that hysteresis and current decrease are caused by surface contamination. These two features are well explained by the equivalent circuit model, reported by Hirao and Oyama (1972), as shown in Figure 6 [13].

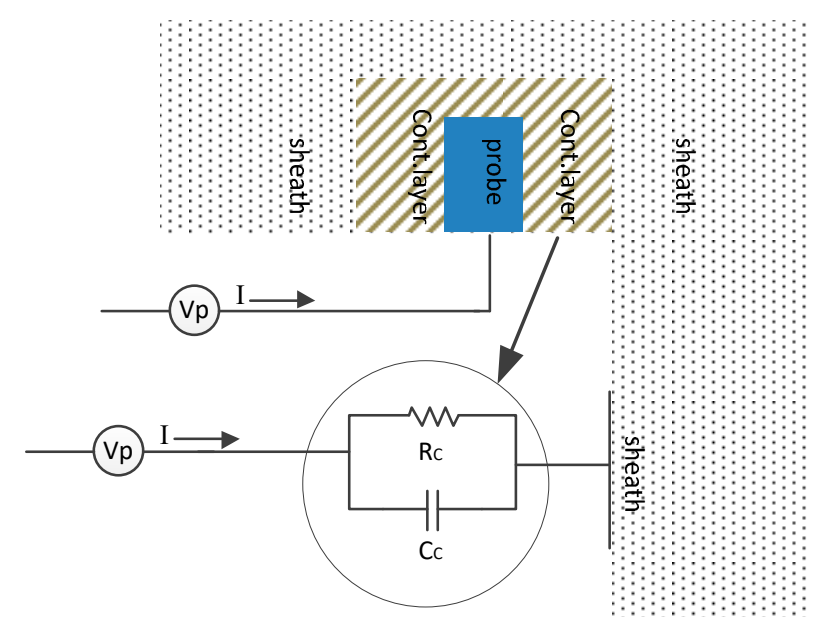

Figure 6. Equivalent circuit of the electrode contamination.

Figure 6 shows that the contamination layer on the probe surface might cause the capacitor Cc and the resister Rc. The Cc and Rc make the hysteresis and current decreased. Therefore, if the power supply of the Langmuir probe is changed from AC to DC, the effect of the capacitor Cc can be counteracted. Loading DC supply on the Langmuir probe and sweeping the voltage from $-60 \mathrm{~V}$ to $+60 \mathrm{~V}$, the I-V curve can be obtained, as shown in Figure 7.

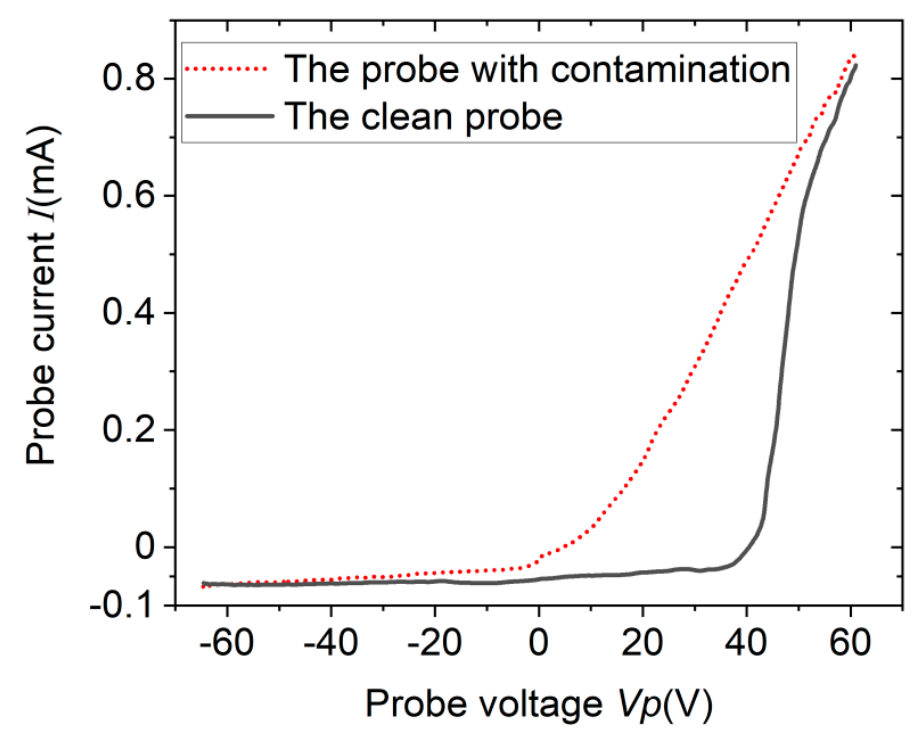

Figure 7. The I-V curve of the clean Langmuir probe and the probe with contaminations driven by DC supply.

Although the effect of capacitance is eliminated, resistance will still cause erroneous information. It is well known that the contamination attached to the probe surface leads to a higher incorrect electron temperature.

From Figure 7, it can be seen that there is no significant change in ion saturation current. Other studies on probes also show that the contamination on the probe has little effect on ion saturation current $[7,8,13]$. When the voltage is less than $-48 \mathrm{~V}$, the ion current of the probe with contaminations 
enters the ion-electron saturation region. The average current in this region is $0.0601 \mathrm{~mA}$ and is close to the ion saturation of the clean Langmuir probe.

The probe surface cleaning can be done in several ways, such as ion bombardment, warm probe, and emissive probe [17-19]. However, most of these methods need to interrupt the plasma reaction. Even the probe has been cleaned, the probe also would be contaminated. In the chemical reaction process such as PECVD, the contamination would be in several minutes. From the above presentation, the Isat can also be measured by the probe with contamination. The electron density $n$ in Equation (4) can be calculated from the I-V curve of the probe with contamination, if the electron temperature Tev is measured by optical emission spectrometry (OES).

\section{The Method of Electron Temperature by OES}

Because this coaxial line plasma is discharged in the enclosed cavity, and the gas flow rate is relatively low, it is usually considered to work at local thermodynamic equilibrium (LTE) [20]. In this case, electron temperature $T_{e}$ can be determined by the relative intensity of two or more spectral lines of the atoms, which have the same ionized stage. In LTE condition, the radiation energy density can be expressed as [21]:

$$
\varepsilon_{\mathrm{mn}}=\frac{1}{4 \pi} \mathrm{N}_{\mathrm{m}} \mathrm{A}_{\mathrm{mn}} \mathrm{hv}_{\mathrm{mn}}
$$

where the subscripts $m$ and $n$ denotes the upper level and the lower energy level of the particles, respectively. $\varepsilon_{\mathrm{mn}}$ is the emission coefficients, $\mathrm{N}_{\mathrm{m}}$ is the particles density of upper level, $\mathrm{A}_{\mathrm{mn}}$ is the transition probability. $h v_{m n}$ is the energy of the transition, where $v_{m n}$ is the frequency of the radiant wave. Using the speed of the light $\mathrm{c}$, the wavelength of the emission light $\lambda_{\mathrm{mn}}$ is given by

$$
\lambda_{\mathrm{mn}}=\frac{\mathrm{c}}{\mathrm{v}_{\mathrm{mn}}}
$$

This leads immediately to

$$
\varepsilon_{\mathrm{mn}}=\frac{1}{4 \pi} \mathrm{N}_{\mathrm{m}} \mathrm{A}_{\mathrm{mn}} \mathrm{hc} / \lambda_{\mathrm{mn}}
$$

In complete LTE at the temperature $\mathrm{T}_{\mathrm{e}}(\mathrm{eV})$, Maxwell-Boltzmann statistics must be applied.

$$
\frac{N_{m}}{N_{n}}=\frac{g_{m}}{g_{n}} \exp \left(-\frac{E_{m}-E_{n}}{k T_{e}}\right)
$$

where $\mathrm{g}$ is the statistical weight of the level, $\mathrm{E}$ is the energy of the level in $\mathrm{eV}, \mathrm{k}$ is the Boltzmann constant factor. The emission intensity measured by spectrometer can be written as

$$
\mathrm{I}_{\mathrm{i}}=\varepsilon_{\mathrm{i}} \mathrm{V} \Omega \mathrm{T}_{\mathrm{i}}^{\mathrm{r}} \eta_{\mathrm{i}} \lambda_{\mathrm{i}}
$$

where $V$ is the volume of the measured plasma, $\Omega$ is the acceptance of solid angle, $T_{i}^{r}$ is the transmittance of the plasma, $\eta_{i}$ is the efficiency of the spectrometer. The emission coefficients of the two spectral lines can be expressed by the following formula according to Equation (10); hence, the spectral is measured with a fixed position and the same spectrometer [22].

$$
\frac{\mathrm{I}_{1}}{\mathrm{I}_{2}}=\frac{\varepsilon_{1}}{\varepsilon_{2}}=\frac{\lambda_{2} \mathrm{~A}_{1} \mathrm{~g}_{1}}{\lambda_{1} \mathrm{~A}_{2} \mathrm{~g}_{2}} \exp \left(\frac{\mathrm{E}_{2}-\mathrm{E}_{1}}{\mathrm{kT} \mathrm{T}_{\mathrm{e}}}\right)
$$

The subscript denotes the chosen spectrum lines. Take the natural logarithm of both sides of Equation (10) leads immediately to

$$
\ln \left(\frac{\mathrm{I}_{1} \lambda_{1} \mathrm{~A}_{2} \mathrm{~g}_{2}}{\mathrm{I}_{2} \lambda_{2} \mathrm{~A}_{2} \mathrm{~g}_{2}}\right)=-\frac{\mathrm{E}_{1}-\mathrm{E}_{2}}{\mathrm{kT} \mathrm{T}_{\mathrm{e}}}
$$


Since multiple spectral lines are used to improve accuracy, taking only one spectral line, Equation (11) can be written as:

$$
\ln \left(\frac{\lambda_{\mathrm{i}} \mathrm{I}_{\mathrm{i}}}{\mathrm{A}_{\mathrm{i}} \mathrm{g}_{\mathrm{i}}}\right)=-\frac{1}{\mathrm{kT}_{\mathrm{e}}} \mathrm{E}_{\mathrm{i}}+\mathrm{C}
$$

where $C$ is a constant. Through measurements and NIST atomic spectra database [23], all values except Te.oes are known. Substituting the adopted spectral lines into Equation (12), by fitting the curve's slope, the electron temperature Tev.oes can be calculated.

The spectrum of this coaxial line plasma is shown in Figure 8, which is measured under the argon fill pressure $\mathrm{Pa}=80 \mathrm{~Pa}$ and the microwave power Mpower $=800 \mathrm{~W}$.

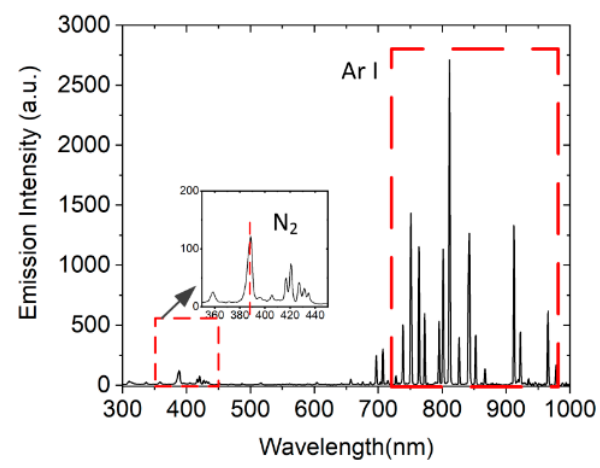

Figure 8. The spectrum of the coaxial line plasma driven by $800 \mathrm{~W}$ microwave at the pressure of $80 \mathrm{~Pa}$.

The spectrum indicates two kinds of particles, nitrogen molecule $\left(\mathrm{N}_{2}\right)$ and Ar. $\mathrm{N}_{2}$ comes from air residue. The air residue is also one of the important causes of probe contamination. Compared with the nitrogen molecule, the peak of this spectrum of Ar I is very obvious with high intensity, which improves the accuracy of measurement of Tev.oes. Although there are many spectral lines of Ar, this does not mean that all spectral lines are suitable for calculating electron temperature, because some spectral lines have a superposition of other spectral lines with a different wavelength, which is very close; besides, the presence of Stark broadening and Doppler broadening would decrease accuracy. Therefore, those spectral lines with a smaller FWHM should be selected. The parameters required by the selected spectrum lines in this article are given in Table 2.

Table 2. Spectroscopic data of chosen lines for determination of electron temperature [23].

\begin{tabular}{cccc}
\hline $\boldsymbol{\lambda i} \mathbf{( n m})$ & $\mathbf{A i}\left(\mathbf{s}^{\mathbf{- 1}}\right)$ & $\mathbf{g i}$ & $\mathbf{E k i}(\mathbf{e V})$ \\
\hline 706.7217 & $3.80 \times 10^{6}$ & 5 & 13.30223 \\
763.5105 & $2.45 \times 10^{7}$ & 5 & 13.17178 \\
772.4207 & $1.17 \times 10^{7}$ & 3 & 13.32786 \\
801.4785 & $9.30 \times 10^{6}$ & 5 & 13.09487 \\
866.7943 & $2.43 \times 10^{6}$ & 3 & 13.15314 \\
912.2967 & $1.89 \times 10^{7}$ & 3 & 12.90702 \\
922.4498 & $5.00 \times 10^{6}$ & 5 & 13.17178 \\
978.4502 & $1.47 \times 10^{6}$ & 5 & 13.09487 \\
\hline
\end{tabular}

Using the peak value of the normalized intensity in Figure 9 and the parameters in Table 2, the value of the specified spectral line can be calculated. Draw multiple points and perform linear regression; the line's slope can be used to calculate the electron temperature. The result is presented in Figure 10 and is determined to be $1.452 \mathrm{eV}$ in this configuration. If the electron temperature Tev.oes $=1.452 \mathrm{eV}$ obtained by OES and the ion saturation current Isat $=0.0601 \mathrm{~mA}$ obtained by the probe with contamination is taken into Equation (4), the electron density can be obtained, which is $n=1.2839 \times 10^{16} \mathrm{~m}^{-3}$. 


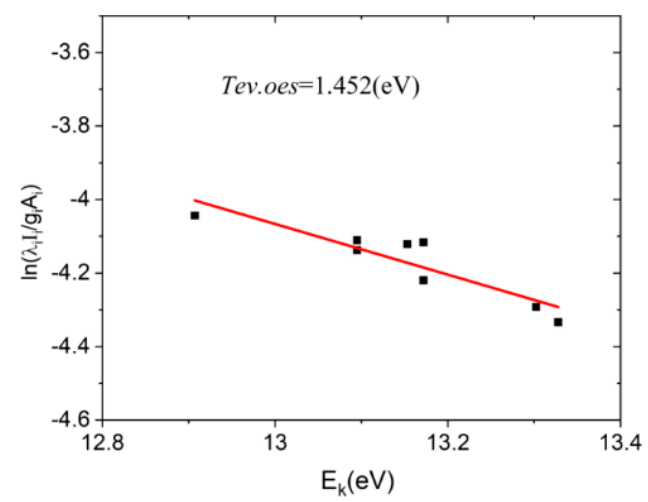

Figure 9. Liner fit for data of specified spectral lines driven by $800 \mathrm{~W}$ microwave at the pressure of $80 \mathrm{~Pa}$.
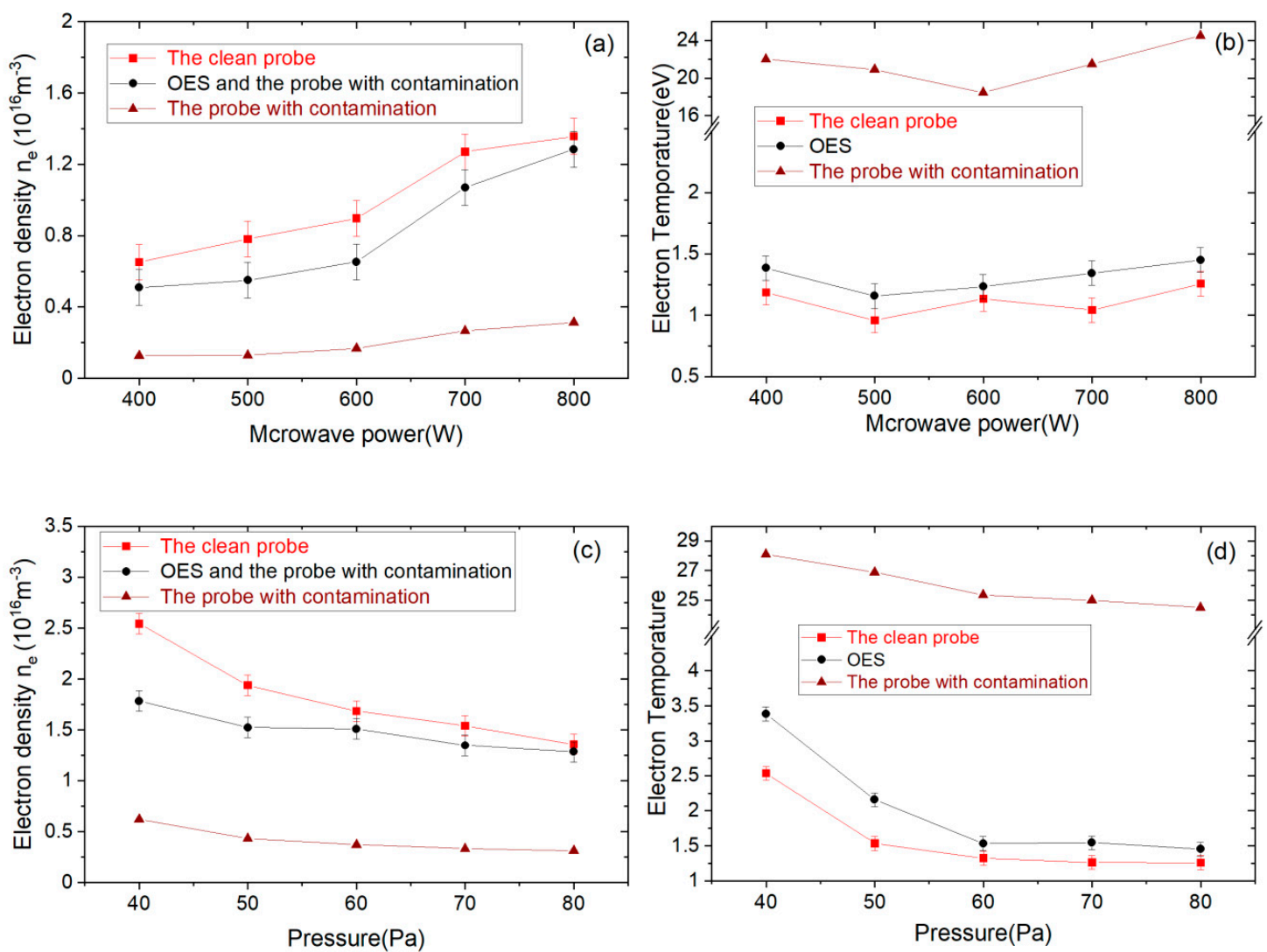

Figure 10. The microwave power and pressure against the electron density and temperature measured by the probe and optical emission spectrometry (OES). $(\mathbf{a}, \mathbf{b})$ The microwave power changes at the pressure of $80 \mathrm{~Pa},(\mathbf{c}, \mathbf{d})$ the pressure changes at the microwave power of $800 \mathrm{~W}$.

\section{Result and Conclusions}

To confirm the reliability of the Langmuir probe and OES, experiments were carried out at different microwave powers and different air pressures. The electron temperature was measured by the clean probe, the probe with contamination, and optical emission spectroscopy method. The electron density was measured by the clean probe, the OES(contribute the Tev.oes), and the probe with contamination(contribute the Isat). The result is shown in Figure 10.

Figure 10 shows that the contamination attached to the probe surface leads to higher incorrect electron temperatures. The electron temperatures obtained by the probe with contamination are about $20 \mathrm{eV}$ higher than the temperatures obtained by the clean probe. When the higher incorrect electron 
temperatures are used to calculate the density, the result will be significantly less than the correct electron density. Comparing the temperature obtained by OES, the clean probe, and the probe with contamination, the temperature Tev.oes obtained by OES is much closer to Tev.probe obtained by the clean probe than by probe with contamination. The Tev.oes is slightly higher than that Tev.probe obtained by the clean probe, which is maybe caused by the OES measurements only presenting the temperature of a part of electrons, especially the high-energy electrons [20]. The difference between these two electron temperatures decreases as the temperature drops. When the electron temperatures are lower than $1.5 \mathrm{eV}$, Tev.oes is basically equal to Tev.probe. Therefore, in the low-temperature plasma, especially below $1.5 \mathrm{eV}$, the electron temperature obtained by OES is accurate. For the high-temperature plasma, the electron temperature obtained may be higher than the correct electron temperature. Moreover, when the Tev.oes is brought into Equation (4), the slightly lower density is obtained in the same Isat. Using this electron temperature, Tev.oes can obtain the approximate electron density from the I-V curve of the Langmuir probe with contamination.

Figure 10 also shows that the electron density is increased with improving microwave power; meanwhile, the electron temperature does not change significantly. Variation of electron density and temperature with pressure are also presented. The results show that when the pressure increases, both the electron density and temperature are decreased.

This paper reports that a simple method to solve the contamination is attached to the probe surface, as the plasma reaction progresses. The electron temperature Tev and electron density $n$ are important parameters in plasma diagnosis. The contamination on the probe surface leads to a higher electron temperature than the correct value. Then, the correct electron density cannot be obtained. The optical emission spectrometry (OES) can be used to solve this problem by obtaining the electron temperature. In addition, the electron temperature obtained by OES spectra can be used to calculate the electron density in the contaminative Langmuir probe I-V curve. The electron temperature and density in different pressure (40-80 Pa) and microwave power $(400-800 \mathrm{~W})$ are measured. It shows that this electron temperature and density obtained by this method are close to the clean probe. Therefore, the contaminative Langmuir probe with the OES can give the approximate electron temperature and density, and the cleaning interval of the Langmuir probe could be significantly extended.

Author Contributions: C.C. and W.F. contributed to the overall study design, analysis, and writing of the manuscript. C.Z., D.L., M.H., and Y.Y. provided technical support and revised the manuscript. All authors have read and agreed to the published version of the manuscript.

Funding: This work was supported by the National Key Research and Development Program of China under 2019YFA0210202, the National Natural Science Foundation of China under Grant 61971097, the Sichuan Science and Technology Program under Grant 2018HH0136, and the Terahertz Science and Technology Key Laboratory of Sichuan Province Foundation under Grant THZSC201801.

Conflicts of Interest: The authors declare no conflict of interest.

\section{References}

1. Liehr, M.; Dieguez-Campo, M. Microwave PECVD for large area coating. Surf. Coat. Technol. 2005, 200, 21-25. [CrossRef]

2. Gosar, Ž.; Đonlagić, D.; Pevec, S.; Kovăc, J.; Mozetǐc, M.; Primc, G.; Vesel, A.; Zaplotnik, R. Deposition Kinetics of Thin Silica-Like Coatings in a Large Plasma Reactor. Materials 2019, 12, 3238. [CrossRef] [PubMed]

3. Cole, M.T.; Milne, W.I. Plasma Enhanced Chemical Vapour Deposition of Horizontally Aligned Carbon Nanotubes. Materials 2013, 6, 2262-2273. [CrossRef] [PubMed]

4. Šamara, V.; Booth, J.; Marneffe, J.D.; Milenin, A.P.; Brouri, M.; Boullart, W. A dc-pulsed capacitively coupled planar Langmuir probe for plasma process diagnostics and monitoring. Plasma Sources Sci. Technol. 2012, 21, 65004. [CrossRef]

5. Chen, F.F. Langmuir probe diagnostics. In Proceedings of the 2003 IEEE-International Conference on Plasma Science (ICOPS), Jeju, Korea, 2-5 June 2003. 
6. Regodón, G.F.; Díaz-Cabrera, J.M.; Fernández Palop, J.I.; Ballesteros, J. Influence of the Ion Mass in the Radial to Orbital Transition in Weakly Collisional Low-Pressure Plasmas Using Cylindrical Langmuir Probes. Appl. Sci. 2020, 10, 5727. [CrossRef]

7. Cherrington, B.E. The Use of Electrostatic Probes for Plasma Dianostics-A Review. Plasma Chem. Plasma Process. 1982, 2, 113-140. [CrossRef]

8. Abe, T.; Oyama, K. An Introduction to Space Instrumentation, 1st ed.; Terra Pub: Okusawa, Tokyo, 2013.

9. Naz, M.Y.; Shukrullah, S.; Ghaffar, A.; Rehman, N.U.; Khan, Y. Test of a new tip material for Langmuir probe diagnostic. J. Appl. Mech. Tech. Phys. 2016, 57, 211-218. [CrossRef]

10. Kau, L.; Huang, H.; Chang, H.; Hsieh, Y.; Lee, C.; Fuh, Y.; Li, T.T. Correlation of Impedance Matching and Optical Emission Spectroscopy during Plasma-Enhanced Chemical Vapor Deposition of Nanocrystalline Silicon Thin Films. Coatings 2019, 9, 305. [CrossRef]

11. Strutynski, C.; Teulé-Gay, L.; Danto, S.; Cardinal, T. Optical Emission Detector Based on Plasma Discharge Generation at the Tip of a Multimaterial Fiber. Sensors 2020, 20, 2353. [CrossRef] [PubMed]

12. Tu, X.; Chéron, B.G.; Yan, J.H.; Cen, K.F. Electrical and spectroscopic diagnostic of an atmospheric double arc argon plasma jet. Plasma Sources Sci. Technol. 2007, 16, 803-812. [CrossRef]

13. Irao, K.; Oyama, K. A Cirtical Study on the Realiability of Electron Temperature Measurements with a Langmuir Probe. J. Geomagn. Geoelectr. 1972, 24, 415-427.

14. Riemann, K.-U. The Bohn sheath criterion and sheath formation. J. Phys. D 1991, 24, 493-518. [CrossRef]

15. Severn, G.D. A note on the plasma sheath and the Bohm criterion. Am. J. Phys. 2007, 75, 92-94. [CrossRef]

16. Merlino, R.L. Understanding Langmuir probe current-voltage characteristics. Am. J. Phys. 2007, 75, 1078-1085. [CrossRef]

17. Strele, D.; Koepke, M.; Schrittwieser, R.; Winkler, P. Simple heatable Langmuir probe for alkali plasmas. Rev. Sci. Instrum. 1997, 68, 3751-3754. [CrossRef]

18. Amatucci, W.E.; Koepke, M.E.; Sheridan, T.E.; Alport, M.J.; Carroll, J.J. Self-cleaning Langmuir probe. Rev. Sci. Instrum. 1993, 64, 1253-1256. [CrossRef]

19. Kalčík, J. Application of warm langmuir probe in the plasma measurements inside plasma-chemical. Czechoslov. J. Phys. 1995, 45, 241-248. [CrossRef]

20. Qayyum, A.; Deeba, F.; Ahmad, S.; Ahmad, Z.; Naseer, M.U.; Shah, S.I.W.; Hussain, S.; Zakaullah, M. Optical Emission and Langmuir Probe Diagnostic Measurements in DC Electrode Pulse Discharge in Nitrogen. High Temp. 2019, 57, 821-831. [CrossRef]

21. Griem, H.R. Plasma Spectroscopy; McGraw-Hill: New York, NY, USA, 1964.

22. Hao, X.; Song, G. Spectral Analysis of the Plasma in Low-Power Laser/Arc Hybrid Welding of Magnesium Alloy. IEEE Trans. Plasma Sci. 2009, 37, 76-82.

23. National Institute of Standards and Technology Data Base. Available online: http://physics.nist.gov/ PhysRefData/ASD/lines_form.html (accessed on 9 October 2020).

Publisher's Note: MDPI stays neutral with regard to jurisdictional claims in published maps and institutional affiliations.

(C) 2020 by the authors. Licensee MDPI, Basel, Switzerland. This article is an open access article distributed under the terms and conditions of the Creative Commons Attribution (CC BY) license (http://creativecommons.org/licenses/by/4.0/). 